\title{
Antimicrobial function for IL-22
}

Two recent studies describe new roles for interleukin-22 (IL-22), an IL-10-family cytokine that is mainly produced by $\mathrm{T}$ helper 17 $\left(\mathrm{T}_{\mathrm{H}} 17\right)$ cells, in host defence at mucosal surfaces.

Aujla et al. explored the role of IL-22 in a mouse model of pneumonia caused by infection with the Gram-negative bacterium Klebsiella pneumoniae. Il22 mRNA could be detected in lung tissue as early as 6 hours after infection with K. pneumoniae, and IL-22 protein levels after 16 hours were higher in infected mice than in uninfected mice. As expected, the IL-22 was shown to be of T-cell origin. Neutralization of IL-22 with specific antibody during infection had a dramatic effect, such that the mice succumbed to bacterial dissemination to the spleen after just 24 hours. By contrast, mice deficient in IL-17, also produced by $\mathrm{T}_{\mathrm{H}} 17$ cells, succumbed to the infection after 48 hours, implying that in this model IL-22 is more important for host defence than IL-17.

Consistent with a role in host defence, IL-22 was shown to increase the proliferation and transepithelial resistance of primary bronchial epithelial cells in vitro. In addition, microarray analysis showed that several host defence genes were upregulated in mouse

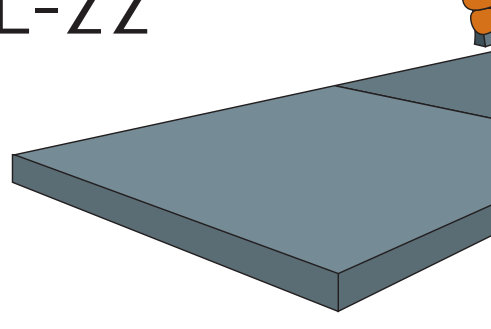

tracheal epithelial cells (MTECs) treated with IL-22 and IL-17 compared with controls. One of these, lipocalin 2, which limits bacterial growth by sequestering iron, proved to be of most interest because IL-22induced killing of K. pneumoniae was significantly reduced in cultures of MTECs derived from lipocalin-2deficient mice compared with wild-type controls.

Zheng et al. studied an enteric bacterial pathogen that infects mice, Citrobacter rodentium, as a model of human infection with enterohaemorrhagic Escherichia coli, and they describe another type of antimicrobial protein that is induced by IL-22. Similar to K. pneumoniae infection, IL-22 production was rapidly induced in the colon in response to C. rodentium infection. Moreover, this early IL-22 production, which required IL-23 and seemed to be derived from dendritic cells rather than T cells, was crucial for protecting mice from continued weight loss and eventual death after $C$. rodentium infection. Indeed, deficiency of IL-22 led to 